\title{
Voyage dans la culture indienne à travers quelques échantillons du cinéma bollywoodien
}

Wendy Cutler, Université Catholique de l'Ouest, Angers, LEMIC / CRILA, France

\section{Introduction}

Cet article portera sur la notion de médiation culturelle qui, dans le cas du cinéma bollywoodien, permet la rencontre de codes cinématographiques, langagiers et culturels propres à la culture indienne, et d'autres issus de la mondialisation du langage filmique actuel.

L'utilisation de ces codes permet de réunifier les différentes facettes de la culture indienne et favorise les échanges tant artistiques qu'économiques et culturels entre l'Inde et les autres pays. D'ailleurs, comme le fait remarquer Jacques Aumont, si le langage cinématographique actuel est dominé par son caractère unitaire et mondialisé, on note cependant que l'Inde produit des films basés pour l'essentiel sur des référents culturels et mythologiques qui lui sont spécifiques. Pour les spectateurs non coutumiers de la culture indienne, les films bollywoodiens semblent difficilement accessibles. Pourtant, ce cinéma connait un succès international grandissant $d u$ fait qu'il fasse appel à un langage cinématographique universel.

L'objectif de cet article sera de voir en quoi cette catégorie de films constitue un véritable outil de communication, ainsi qu'un portail ouvrant sur la culture de l'Autre. Pour ce faire, nous verrons que si les cinéastes de tous pays basent souvent leurs scénarios sur la mythologie, cela est d'autant plus vrai des réalisateurs indiens. Ces derniers, en effet, s'inspirent largement de la mythologie hindoue - l'hindouisme étant, en Inde, suivi par près de $80 \%$ de fidèles. Au niveau des représentations visuelles, l'hindouisme occupe également une place prépondérante avec de nombreux temples dédiés aux divinités, des peintures, sculptures, danses sacrées, pièces de théâtre et depuis plus d'un siècle, l'apparition d'un $7^{\mathrm{e}}$ Art.

\section{Le cinéma indien}

Dès son arrivée, les Indiens se sont vite approprié cette nouveauté occidentale que constituait le cinéma. En effet, seulement six mois après la première projection du cinématographe en France, Maurice Sestier, l'un des opérateurs des frères Lumière, débarqua 
à Bombay pour y organiser la première projection en Inde. On comprit rapidement qu'il fallait chercher des films inédits et accessibles au large public et qu'avant tout, il fallait trouver des images issues de la culture indienne. Le culte de l'image en Inde faisant partie de la tradition depuis des millénaires, il fallait adapter les images animées pour satisfaire la demande. Très vite, le genre mythologique se développa grâce à Dhundiraj Govind Phalke (1870-1944), plus connu sous le nom de Dadasaheb Phalke ou encore le «père du cinéma indien $»$ (Thoraval 21).

L'appropriation du cinéma en Inde peut être expliquée en partie grâce au principe de la maya, telle qu'elle est définie dans l'hindouisme. La maya, qui signifie «illusion », est symbolisée par un miroir et correspond au monde des apparences, au pouvoir des artifices. Dans la définition qu'il donne de ce terme, Heinrich Zimmer nous dit : « La maya est "l'art" : ce par quoi un artifice, une apparence, est produit. Maya est précisément le pouvoir ou l'art du créateur. En d'autres termes, la "magie" selon Jacob Boehme »" (24). Nous pouvons donc souligner la ressemblance entre la maya et le cinéma. En effet, celui-ci à ses débuts exerçait une magie particulière sur les spectateurs, en particulier avec l'invention par Georges Méliès des premiers trucages. Le cinéma accordait ainsi une place essentielle à l'illusion et pouvait tromper l'œil humain, tout comme la maya en Inde.

Avec le cinéma indien, nous sommes ainsi dans un cas typique de langage gardant ses racines (par un rattachement permanent aux traditions), tout en s'ouvrant au monde. Cette rencontre des cultures et des langages artistiques permet une sorte de syncrétisme, concrétisé par l'émergence d'un nouveau genre de cinéma. Dans ce dernier, se mêlent à la fois toutes les richesses des cultures orientales et occidentales.

En ce sens, le cinéma ouvre la porte à l'altérité : l'altérité intra-culturelle spécifique à l'Inde (puisque dans ce pays, il existe plusieurs religions mais aussi plusieurs langues), et l'interculturalité au niveau mondial. Ce n'est d'ailleurs pas étonnant que le nom donné au cinéma populaire indien soit issu d'un néologisme entre Bombay et Hollywood.

Afin d'illustrer nos propos par des exemples concrets, nous proposons de nous appuyer sur nos travaux de thèse, basés sur le cinéma bollywoodien des années 1970-80. Nous travaillerons ainsi sur une sélection de films qui constituent un exemple de réactualisation de mythes anciens issus des deux poèmes épiques que sont le Mahabharata et le Ramayana. 


\section{Mythologie et cinéma}

Dans cette étude, la mythologie nous servira de fil conducteur nous permettant de déchiffrer les codes spécifiques du cinéma bollywoodien. Depuis son apparition en Inde, le cinéma n'a pas cessé de puiser dans les nombreux récits mettant en scène des divinités, et ce, afin d'enrichir, voire de «déifier» les représentations filmiques. En reprenant les propos de M. K. Raghavendra, spécialiste indien du cinéma, nous constatons que : « La structure du cinéma populaire indien a également été définie, de manière utile, "comme un palimpseste capable de s'étendre et de se répéter jusqu'à l'infini” avec comme texte précurseur le Ramayana/Mahabharata $»^{2}$ (13). Ces deux poèmes épiques que sont le Ramayana et le Mahabharata ${ }^{3}$ servent en effet de base scénaristique à de nombreux films indiens du fait qu'ils regorgent de mythes, de personnages divins, d'actes héroïques, de démons, de conflits et de batailles.

Toutefois, il est important de noter qu'avec le cinéma bollywoodien, nous ne sommes pas en présence d'une porte béante, laissant s'échapper la culture d'un pays pour en absorber une autre : il est question de porte d'échanges, de mélange des genres et de syncrétisme culturel avec création d'un nouveau genre artistique.

Puisqu'il est question de la réactualisation de mythes anciens, notons d'ores et déjà que le célèbre mythologue américain, Joseph Campbell, propose dans la théorie du «Voyage du Héros », un schéma narratif archétypal que l'écrivain dégage de l'étude de différentes mythologies. Il se focalise ainsi sur les grandes mythologies du monde entier, d'où le terme de «monomythe » qui évoque l'universalité du mythe. Ce qui prouve que la mythologie peut, en elle-même, constituer une porte d'échanges entre les grandes civilisations.

À ce stade, nous pouvons souligner un point important : le cinéma bollywoodien n'est pas représentatif du cinéma indien en général. Il ne constitue qu'un genre parmi d'autres et s'adresse à « un » public en particulier. L'Inde est un pays multilingue, comptabilisant, selon le linguiste québécois Jacques Leclerc, plus de 4000 langues et dialectes. Ce pourquoi il faut bien faire la distinction entre le cinéma bollywoodien et les différents types de cinémas indiens, comprenant des films tournés en langues régionales un peu partout dans le pays.

Au niveau du langage cinématographique, nous pouvons constater que derrière le cinéma bollywoodien, qui a avant tout une fonction de divertissement, se trouve un puissant outil de communication reposant sur des codes. En Inde, on considère les années 1950-65 comme l'âge d'or du cinéma indien et cette période vit l'émergence de certains codes et thématiques comme la pureté des héros, l'exaltation de la famille, le respect de la religion, le 
manichéisme, la durée des films (2h30-3h minimum) et le nombre de danses et de chants (six minimum par film, le record étant de soixante-dix chansons dans un seul film). Dès les débuts du cinéma indien, la danse et le chant faisaient partie intégrante des films, perpétuant cette tradition des arts sacrés tout en les adaptant au langage filmique. En effet, les danses bollywoodiennes aujourd'hui constituent un mélange de danses classiques et traditionnelles avec des éléments populaires. Certains films rendent même un hommage aux danses sacrées, tel Devdas (2003) qui brille par les nombreuses séquences de Bharata Natyam et Kathak.

Ainsi il existe des codes propres aux films bollywoodiens, néanmoins certains de ces codes sont universels et touchent, par exemple, la manière de montrer la peur ou le danger. Nous retrouvons également ces codes à travers l'utilisation de symboles pouvant être interprétés par plusieurs civilisations.

\section{L'utilisation des symboles}

Notre premier exemple de symbole à caractère « universel » est celui de la chaine, utilisé dans le film Zanjeer (1973). Il s'agit d'un symbole fort qui apparait tout au long du film. L'histoire commence par le meurtre des parents du héros, qui se prénomme Vijay. D’entrée, nous voyons que la chaine parentale est rompue. « D’une façon générale, la chaine est le symbole des liens de communication, de coordination, d'union [...]. Dans un sens socio-psychologique, la chaine symbolise la nécessité d'une adaptation à la vie collective et la capacité d'intégration au groupe » (Chevalier et Gheerbrant 200).

Dans le film, le spectateur découvre qu'après la disparition de ses parents, le jeune Vijay se tient à l'écart de ses camarades. C'est donc la chaine sociale, après la chaine parentale, qui est brisée. L'enfant ne parvient pas à s'intégrer à un groupe. Et tout au long du film, Vijay apparaitra tel un personnage solitaire. Pour rétablir la communication, il devra trouver le maillon manquant et «réparer» la chaine. Ce qui équivaudra à trouver le responsable et venger la mort de ses parents.

Le symbole de la chaine se retrouve dans de nombreux films occidentaux et asiatiques, comme La chaine (1958) de Stanley Kramer, mettant en scène deux évadés de prison qui doivent survivre en étant attachés l'un à l'autre par une chaine. Ils se retrouvent liés à jamais - même lorsque la chaine est brisée. Ou encore dans le film japonais Dolls (2002) de Takeshi Kitano, qui met en scène des amants attachés symboliquement par une corde rouge. 
L'arme blanche est également un symbole très usité du cinéma indien. Dans le film Nastik (1983), le héros, qui se prénomme Shankar, assiste à l'assassinat par arme blanche de son père lorsqu'il n'est encore qu'un enfant. Shankar n'a alors comme seul objectif que de venger la mort de son père. Il souhaite que justice soit faite et il le dit clairement à la divinité Krishna : «Je me ferai justice moi-même. Mon père innocent a été tué avec ce couteau. Ce couteau décidera du destin du propriétaire » ${ }^{4}$ - le propriétaire (landlord) étant « le méchant ».

«Le symbole du couteau est fréquemment associé à l'idée d'exécution judiciaire, de mort, de vengeance, de sacrifice [...]. Le couteau est l'instrument essentiel des sacrifices, en de nombreuses épreuves initiatiques, à commencer par la circoncision » (Chevalier et Gheerbrant 306). Lorsqu'il est question de sacrifice, nous pouvons bien évidemment faire référence à l'épisode biblique dans lequel Abraham doit - sur ordre divin - tuer son fils Isaac avec un couteau.

Dans Nastik, lorsque le héros annonce au dieu Krishna qu'il va venger la mort de son père avec le couteau ayant servi à tuer ce dernier, on assiste à une scène qui marque le passage de l'enfance à l'âge adulte. Mais pour passer à l'âge adulte, Shankar doit subir une épreuve initiatique. Le couteau devient alors le symbole de la vengeance, mais également l'instrument qui va projeter le héros dans un monde inconnu et faire de lui un homme. En effet, armé de ce couteau, il va se rendre dans la chambre où dort le meurtrier de son père pour le tuer. Lors du combat qui suit, Shankar réussit à lui crever un œil et devient ainsi un homme.

Le couteau est également un symbole fort dans le film Zanjeer, mentionné précédemment. Le personnage féminin de Mala est introduit à l'image par le biais d'une chanson dans laquelle elle met en avant son habilité à manier les couteaux. Le fait que ce soit une femme qui manie aussi habilement cette arme souligne l'idée de la menace de castration pesant sur les hommes. À l'image d'une femme forte, n'ayant besoin de personne, Mala tient tête aux hommes. En effet, lorsqu'un homme lui fait des avances, elle n'hésite pas à le menacer avec son couteau. En cela, le couteau acquiert une valeur quasi phallique qui permet à Mala d'intimider les hommes.

Dans les films indiens que nous avons choisi d'étudier, un autre symbole représente le passage de l'enfance à l'âge adulte : c'est celui du train. Dans le film Coolie (1983), le jeune héros, prénommé Iqbal, est séparé de ses parents suite à un déluge qui a ravagé son village. Un jour, le jeune garçon aperçoit sa mère à bord d'un train. Celle-ci, qui a perdu la mémoire, 
ne le reconnait pas. Nous voyons alors le héros courir après le train en appelant sa mère dans l'espoir qu'elle le reconnaisse. Mais le train finit par emmener sa mère loin de lui.

Le départ du train peut symboliser le début d'une nouvelle aventure, le commencement d'un voyage - aussi bien pour le héros qui voudra retrouver sa mère que pour la mère elle-même qui finira par retrouver la mémoire. L'image de l'enfant courant après le train représente son incapacité à contrôler la situation. Il n'est pas à bord du train mais regarde, impuissant, celui-ci s'éloigner. Alors que le réseau de chemin de fer est une organisation ponctuelle représentant l'ordre et un fonctionnement à la minute près, dans cette scène de départ, nous constatons que le héros échappe à son destin. Nous pouvons ajouter que le réseau ferroviaire " permet toutes les communications et tous les échanges » (Chevalier et Gheerbrant 961). En cela, ce départ symbolise - tout comme la métaphore de la chaine dans Zanjeer - une rupture dans la vie du jeune garçon. Il devra rétablir la communication et retrouver sa mère.

Dans Coolie l'action se situe principalement dans la gare où le jeune héros trouve un travail en tant que bagagiste. Par la suite, le spectateur découvre un Iqbal devenu adulte au moment où un train arrive en gare. Le train sert donc d'ellipse au film puisque nous découvrons - juste après son arrivée - le héros une fois adulte, incarné par l'acteur Amitabh Bachchan.

Un autre film indien qui utilise le train comme ellipse est Nastik. Lorsque le jeune Shankar s'enfuit après avoir blessé le propriétaire, il saute sur le toit d'un train en marche pour échapper à ses poursuivants. Le train est alors un élément positif, remplissant sa fonction première : celle de moyen de transport. Le train servira donc d'échappatoire pour le héros qui saute. Le réalisateur effectue alors un arrêt sur image où l'on voit le jeune garçon suspendu en l'air, juste avant qu'il n'atterrisse.

Dans le film Laawaris (1981), le train est également énonciateur du début du voyage. En effet, lorsque le héros - qui n'est encore qu'un bébé - est confié à Gangu, celui-ci l'emmène à bord d'un train. L'enfant quitte alors ses proches, en particulier son père qu'il retrouvera à la fin du film, et ce voyage symbolise le début d'une nouvelle vie. Il s'agit, en d'autres termes, d'une rupture avec ses origines que l'enfant passera sa vie à tenter de retrouver.

Un autre genre de film mettant en scène les chemins de fer est bien évidemment le western américain, ce qui montre bien que le cinéma en général utilise les mêmes codes, pouvant être déchiffrés par un large public. Dans les westerns, le chemin de fer représente la 
Révolution industrielle, le progrès technique et la richesse. En effet, il est courant qu'un train soit détourné par des bandits dans le but de voler le butin qui s'y trouve. Parfois, l'emplacement même d'une gare en construction constitue l'enjeu de tout le film (comme dans Il était une fois dans l'Ouest). Dans certains films indiens, tout comme dans les westerns, ce moyen de transport révolutionnaire à tout point de vue représente également l'arrivée d'un nouveau personnage dans une ville ou un changement significatif. Il est donc un élément annonciateur de changement, de nouveauté mais aussi d'incertitude.

\section{Le labyrinthe, thème mythologique universel}

Outre ces codes universels qui renvoient à l'unicité du langage cinématographique, les films indiens, en se réappropriant la mythologie hindoue, reprennent également des thématiques mythologiques universelles. À titre d'exemple, nous pouvons évoquer la traversée du labyrinthe qui est un thème central dans un grand nombre de films, aussi bien européens qu'américains ${ }^{5}$. Ce thème n'est pas non plus sans rappeler le mythe grec du Minotaure, enfermé par le roi Minos dans un labyrinthe. Chaque fois, le héros doit subir l'épreuve du labyrinthe avant d'atteindre le but ultime de son voyage.

Nous retrouvons également ce thème universel dans un grand nombre de films indiens, notamment dans le film Deewaar (1975), lorsque le héros est poursuivi par son frère ennemi. En effet, les rues de Bombay deviennent un obstacle pour le héros qui tente de rejoindre le « centre » qui est en fait le temple où se trouve sa mère. La fin du film Muqaddar Ka Sikandar (1978) met également en scène une course poursuite pouvant être rapprochée de la traversée du labyrinthe. Le film Don (1978) fait également référence au labyrinthe. À la fin du film, le héros Vijay poursuit Daljit et ses enfants dans des petites ruelles de la ville. La façon dont les personnages se cachent derrière les murs et tentent d'échapper à Vijay renvoie inéluctablement à un labyrinthe. En effet, les rues se ressemblent et sont ainsi trompeuses. Les murs sont de plus en plus hauts au point que le spectateur n'en voit pas la fin, ce qui donne une illusion d'infinité.

Néanmoins, d'autres codes sont propres à la culture indienne, avec des symboles liés spécifiquement à la mythologie hindoue. Dans Deewaar - film que nous venons de citer - le héros sort blessé par balle du labyrinthe mais parvient tout de même à rejoindre le temple au volant d'une voiture. En arrivant, il s'écrase contre les marches menant au temple. Les roues 
de sa voiture se retrouvent encastrées dans les marches - scène qui renvoie un public averti à la mort du personnage mythologique Karna dans le Mahabharata, cité précédemment.

En effet, l'histoire principale du Mahabharata raconte une guerre divisant les Pandava (qui représentent le bien) et les Kaurava (qui représentent le mal), deux camps issus d'une même famille. Lors de la bataille finale, l'on assiste à la confrontation d'Arjuna et Karna (les deux frères ennemis). Alors que Karna lutte contre Arjuna, la roue de son char s'enfonce dans la terre, et il est impossible de l'extraire. Krishna, le mentor d'Arjuna, lui dit alors: «Je vais te dire ce qui bloque ta roue. C'est la terre elle-même avec ses mains boueuses. Subitement elle prend part à la bataille, elle se défend, elle a décidé de nous aider. C'est elle qui vient de happer la roue de ce char et qui ne la relâchera plus » (Carrière 283). Et c'est à ce moment qu'Arjuna profite de l'immobilisation du char pour tuer son adversaire redoutable.

Ainsi, comme nous le voyons, le char du récit mythologique a été remplacé, dans Deewaar, par une voiture, dans un effort d'adaptation aux réalités contemporaines. Nous pouvons également citer le film intitulé Karan Arjun (1995) qui fait également directement référence au mythe des frères ennemis issu du Mahabharata. Ce constat incite à penser qu'à travers l'étude du cinéma d'un pays, il est possible de découvrir sa culture, son identité et de mesurer l'influence que d'autres pays ont eue sur celui-ci.

\section{La notion d'apprentissage}

Nous venons de voir que les films bollywoodiens constituaient un nouveau mode de représentation des mythes anciens, établissant par là un lien omniprésent avec les mythes fondateurs de cette culture. Le fait que les films bollywoodiens s'enracinent autour de mythes fondateurs permet de réunir les spectateurs autour d'un même fil conducteur qu'est la mythologie (et, plus généralement, la foi). C'est donc à un double niveau que se construit l'apprentissage : au niveau des mythes fondateurs, mais aussi au niveau de leur actualisation, comme nous venons de le voir à travers les différents exemples.

Généralement, les mythes fondateurs trouvent leur équivalent dans les autres mythologies. Ainsi l'étude de ces mythes dans le cinéma indien est à la fois un outil d'apprentissage de l'hindouisme mais aussi un moyen de trouver des passerelles entre les différentes civilisations. C'est, ici, l'aspect éducatif et pédagogique qui est en jeu.

Le fait que la mythologie soit aussi présente dans les films indiens montre deux choses : la première est que les croyances sont toujours très présentes dans ce pays et qu'elles 
constituent un excellent support de communication - même la publicité utilise des symboles mythologiques. En cela, la mythologie donne une certaine portée aux films qui, en contre partie, assurent la diffusion et le maintien des traditions.

La seconde est que les cuvres de fiction font office d'instrument de communication dont la portée dépasse le simple divertissement pour accéder à une dimension politicoculturelle. Ce qui montre qu'en Inde, l'art permet, au-delà du simple récit d'une histoire, de diffuser la culture indienne et, parallèlement, de l'adapter aux gouts du jour, puisque comme nous le voyons dans les films bollywoodiens, les mythes sont souvent adaptés pour être mieux compris par les publics et servir l'intrigue de l'œuvre.

Le fait que l'image soit un outil artistique dont la portée va bien au-delà et touche au politique explique que les films produits par l'industrie cinématographique indienne rendent compte à la fois de la compréhension des mythes indiens par les Indiens eux-mêmes, et donc de la façon dont le pays se voit lui-même, mais également de la manière dont il décide de se montrer aux autres. Cela montre que, pour l'Inde, le cinéma est devenu un outil de communication du pays lui-même vis-à-vis des autres pays.

\section{L'Inde vue par l'Occident}

À ce stade, il est important de souligner que la rencontre des cultures filmiques n'a pas que pour effet de multiplier les échanges. Car derrière l'image, c'est la vision d'un pays et de sa société qui est en jeu. Or l'image n'est pas objective, que ce soit au niveau de l'image que l'Inde veut donner d'elle-même ou de l'image que s'en fait l'Occident.

Ainsi, les œuvres cinématographiques tournées en Inde par des équipes de réalisation étrangères ont-elles pour particularité de donner un aperçu de la vision que les pays étrangers ont de l'Inde - vision parfois considérée comme caricaturale. Cela peut donner lieu à des controverses : nous pensons notamment au succès planétaire de Slumdog Millionnaire, réalisé par Danny Boyle et sorti en 2009. Le film est devenu un récit montrant l'Inde selon l'image que l'Occident (ou tout du moins le cinéaste) se fait de ce pays. Cette œuvre a remporté un véritable succès en Occident et reçu de nombreuses récompenses alors qu'en Inde, il a reçu un accueil plutôt mitigé car les Indiens y voyaient davantage une présentation de l'Inde telle qu' « imaginée par les Blancs » et dans laquelle ils ne se retrouvaient pas. Il est donc question d'image « problématique » ou de « décalage » entre ce qu'un pays souhaite montrer de luimême et la manière dont l'Autre le perçoit. 


\section{Conclusion}

Toutefois, cet exemple ne doit pas faire office de généralité. Au-delà, nous constatons qu'une interdisciplinarité nait du mélange des langages artistiques et culturels propres à chaque pays. Et que l'utilisation des uns permet la diffusion des autres. En cela, le cinéma permet les échanges culturels avec l'extérieur, mais surtout, il permet de donner aux traditions et aux croyances, une continuité qui ne soit pas synonyme de rigidité. Point qui nous permet d'affirmer que, derrière la notion d'échanges culturels, il y a l'idée de syncrétisme au sens où un art à part et à part entière s'est créé de la rencontre de plusieurs cultures.

\section{Bibliographie}

\section{Ouvrages}

Aumont, Jacques, Alain Bergala, Michel Marie et Marc Vernet. Esthétique du film. Paris : Nathan, 1983.

Aumont, Jacques et Michel Marie. L'Analyse des films. Paris : Nathan. 1988.

Bregeat, Raïssa., dir. Indomania, Le cinéma indien des origines à nos jours. Paris : Cinémathèque française, 1996.

Campbell, Joseph. The Hero With a Thousand Faces. Princeton : Princeton UP, 1973 (1949).

Carrière, Jean-Claude. Le Mahabharata. Paris : Belfond, 1989.

Chevalier, Jean et Alain Gheerbrant. Dictionnaire des symboles. Paris : Laffont/Jupiter, 1982 (1969).

Hennebelle, Guy. (dossier réuni par Aruna Vasudev et Philippe Lenglet). CinémAction, Les cinémas indiens, Paris : Cerf, 1984.

Jaffrelot, Christophe. L'Inde contemporaine, De 1950 à nos jours. Paris : Fayard/Ceri, 2006.

Landy, Frédéric. L'Inde ou le grand écart. Paris : Documentation française, 2007.

Leclerc, Jacques. Langue et société. Paris : Mondia, 1986.

Passek, Jean-Loup, dir. Le cinéma indien. Paris : Centre Georges Pompidou, 1983.

Raghavendra, M. K. Seduced by the Familiar, Narration and Meaning in Indian Popular Cinema. Delhi : Oxford UP, 2008.

Thoraval, Yves. Les cinémas de l'Inde. Paris : Harmattan, 1998.

Vogler, Christopher. Le guide du scénariste. La force d'inspiration des mythes pour l'écriture cinématographique et romanesque. Paris : Dixit, 2009. 


\section{DVD}

Zanjeer, de Prakash Mehra. 1973. Mumbai : Prakash Mehra Productions. 132 minutes.

Deewaar, de Yash Copra. 1975. Mumbai : Trimurti Films PVT. LTD. 160 minutes.

Don, de Chandra Barot. 1978. Mumbai : Nariman Films. 175 minutes.

Muqaddar Ka Sikandar, de Prakash Mehra. 1978. Mumbai : Prakash Mehra Productions. 181 minutes.

Laawaris, de Prakash Mehra. 1981. Mumbai : Prakash Mehra Productions. 189 minutes.

Nastik, de Pramod Chakravorty. 1983. Mumbai : Vinod Doshi Productions. 160 minutes.

Coolie, de Manmohan Desai et Prayag Raj. 1983. Mumbai : Ketan Desai Movies. 177 minutes.

Karan Arjun, de Rakesh Roshan. 1995. Mumbai : Eros Entertainment.176 minutes.

Devdas, de Sanjay Leela Bhansali. 2002. Mumbai : Mega Bollywood. 180 minutes.

Slumdog Millionnaire, de Danny Boyle. 2008. Paris : Pathé, Los Angeles : Fox Searchlight

Pictures. 120 minutes.

\footnotetext{
NOTES

${ }^{1}$ " Maya is "art": that by which an artifact, an appearance, is produced. Maya is precisely the maker's power or art. "Magic" in Jacob Boehme's sense $[\ldots]$ ».

${ }^{2}$ « The body of Bombay cinema has also been usefully read [...] "as a palimpsest capable of endless expansion and repetition $[\ldots]$ " with its precursor text being the Ramayana/Mahabharata ».

${ }^{3}$ Le terme Ramayana signifie «L'Histoire du Prince Rama» et le Mahabharata peut etre traduit par «La Grande Histoire de l'Humanité ».

${ }^{4}$ «I will myself do justice. My innocent father was killed with this knife. Now this knife itself will decide the fate of the landlord. »

${ }^{5}$ Pour n'en citer que quelques-uns, Jim Henson a réalisé le film fantastique anglo-américain Labyrinthe, sorti en 1986, qui, comme le titre l'indique, se déroule dans un labyrinthe. Le labyrinthe de Pan, film espagnol de Guillermo del Toro, sorti en 2006, raconte 1'histoire d'une jeune fille qui doit accomplir trois épreuves imposées par le gardien du labyrinthe. Ou plus récemment encore, le film américain de Christopher Nolan, Inception, sorti en 2010, suit des personnages capables de créer des rêves et de les infiltrer. Ariane, l'un des personnages, doit planter le décor du rêve sous forme d'un labyrinthe.
} 